\title{
Geodesics on the momentum phase space with metric ${ }^{C_{g}}$
}

\author{
İsmet Ayhan ${ }^{\mathrm{a}^{*}}$ \\ ${ }^{a}$ Department of Mathematics Educations, Faculty of Education, Pamukkale University, Denizli, Turkey \\ *Corresponding author E-mail: iayhan@ pau.edu.tr
}

\section{Article Info}

Keywords: The Hamilton Mechanic System, Geodesics on The Cotangent Bundle, Space like Surface

2010 AMS: $35 Q 70,53 C 22,53 C 25$

Received: 16 February 2018

Accepted: 5 March 2018

Available online: 11 March 2018

\begin{abstract}
In this paper, a system of the differential equations giving geodesics on the momentum phase space with pseudo Riemann metric ${ }^{C} g$ of a Hamilton space is found by using the Euler Lagrange equations. Then, space like geodesics on pseudo hyperbolic 2-space $H_{1}^{2}$ are obtained. Finally, a system of the differential equations giving geodesics on the cotangent bundle with pseudo Riemann metric ${ }^{C} g$ of $H_{1}^{2}$ is get.
\end{abstract}

\section{Introduction}

The geometry of the cotangent bundle is one of the most important subjects attracted the attention of mathematicians as well as physicists. A Lagrange mechanical system consists of a configuration space which contains the trajectory of each of the moving particles of a system with n-particles and Lagrangian which gives the difference between the kinetic and potential energy at any stage of the each particle in system [1].

The position and velocity coordinates depended on time parameter $\mathrm{t}$ of the motion of the each particle of a system with n-particles are represented by the coordinates of any point on the tangent bundle of a (pseudo) Riemann manifold. Lagrangian is defined as a real valuable and differentiable function on the tangent bundle [7].

The position and velocity coordinates of a moving particle in the system at any instant $t$ are found by the Euler Lagrange equations. The Euler Lagrange equations are second order ordinary differential equations which depend on position and velocity of each particle in system [7]. Gravitational field is given by a (pseudo) Riemann metric. The movement depended on time t of all particles in system by the only effect of the gravitational field is described by a (pseudo) Riemann manifold. The trajectory of a moving particle by the effect of the gravitational field describes a geodesic, the shortest one among the curves passing from one point to another, in a (pseudo) Riemann manifold [8].

In different place of space, the effect of the gravitational field on moving particles is different. As metric is change, (pseudo) Riemann manifold must change. Moreover, in different (pseudo) Riemann manifold, the trajectory of moving particles by the only effect of the gravitational field, geodesics, must change. So, we examined geodesics on different space forms such as $H_{1}{ }^{2}, \mathrm{~S}_{1}{ }^{2}, \mathrm{~S}^{3}$ and its tangent sphere bundle from [[2],[3],[4]].

From the references [1], [5], [7], [8], [9] and [10], we searched some concept such as, the movement of particles in the gravitational field, total energy function in a Hamilton mechanic system, kinetic and potential energies of a moving particle, the conservation of energy and the conservation of momentum.

Then, we found out that how obtained space like, time like and null geodesics of the unit 2-sphere with index one by using the Euler Lagrange equations from [6].

Moreover, we analyzed the general equations of geodesics on the cotangent bundle with pseudo Riemann metric ${ }^{C} g$ which is called as Riemann extension of the symmetric affine connection [11].

In this paper, we defined Lagrangian on the cotangent bundle and we calculated geodesics on the cotangent bundle with a pseudo Riemann metric ${ }^{C} g$ by using the Euler Lagrange equations.

Then, we obtained space-like geodesics on the pseudo hyperbolic 2-space by using the Euler Lagrange equations. Finally, we calculated the general equations of geodesics on the cotangent bundle of pseudo hyperbolic 2-space with the pseudo Riemann metric $C_{g}$. 


\section{The motion of the particles in the Hamilton Space}

In this section, we obtain the geometrical interpretations of some concepts, well known in classic mechanic such as the movement of the particles by the effect of the gravitational field, the conservation of energy and momentum.

In classical mechanic, the motion of a system with n-particles is described by the position coordinates and the momentum coordinates of each of the particles in system. The position coordinates of a particle depend on the position vector in three-dimensional space. The momentum coordinates of a particle depend on a one form associated with the tangent vector of the curve determined by trajectory of the moving particle [10].

If the configuration space represents by an $n=3 \mathrm{~N}$ dimensional manifold whose the local coordinate functions give the positions coordinates of each of the particles at any instant $\mathrm{t}$, the phase space of the configuration space can represent by an $2 \mathrm{n}$ dimensional manifold whose the local coordinate functions give the position and the momentum coordinates of each of the particles at any instant t. In other words, the phase space of the configuration space must represent 2 n-dimensional the cotangent bundle as the configuration space of moving particles by the only effect of the gravitational field represents a n-dimensional differentiable manifold M [10]. The arc length between the position coordinates of infinitely close two points on the trajectory of a particle in the configuration space is determined by

$$
d s^{2}=g_{k j}(x) d x^{k} d x^{j}
$$

where $g_{k j}(x)$ is a pseudo Riemann metric on M. The trajectory of a moving particle in M is represented by a curve $\gamma: I \subset R \rightarrow M$. For any time t, the position coordinates of $\gamma$ is given by $x^{i}(t)=x^{i} \circ \gamma(t), i=1, \ldots, n$ and the velocity and momentum coordinates of $\gamma$ are given by $y^{i}=\frac{d x^{i}}{d t}$, and $p_{i}=g_{i j} y^{j}$, respectively.

The curve $\gamma$ must be called as the space-like, the time-like or the light-like curve if the value under the pseudo Riemann metric $g$ of the unit tangent vector $v$ at every point of $\gamma, d s^{2}=g(v, v)=\varepsilon$ is provided $\varepsilon=1, \varepsilon=-1$ or $\varepsilon=0$, respectively [3].

The sum of the kinetic and the potential energies of all moving particles in the system are represented by the function $H: T^{*} M \rightarrow R$ in the phase space and it is called Hamiltonian.

Assuming that all particles in the system act only the effect of the gravitational field. Then the Hamiltonian $\mathrm{H}$ equals to the sum of the total energies of the moving all particles in system and the Hamiltonian $\mathrm{H}$ of the test particles i.e. unit mass particles are described by

$$
H\left(x^{i}, p_{i}\right)=\frac{1}{2} g^{i j}(x) p_{i} p_{j}
$$

where $g^{i j}(x)$ is a metric tensor with type (2,0) given by $g^{i k} g_{k j}=\delta_{j}^{i}$ [5]. The Hamilton space M consists of n-dimensional differentiable manifold $\mathrm{M}$ and a Hamiltonian $\mathrm{H}$ on the cotangent bundle of $\mathrm{M}$. Assuming that $\eta=p_{i} d x^{i}$ is the basic 1 -form on $\mathrm{T}^{*} \mathrm{M}$. The exterior differential $\vartheta=-d \eta$ of the basic 1-form $\eta$ is the 2-form given by $\vartheta=d x^{i} \wedge d p_{i}$ with respect to the induced local coordinates of $\mathrm{T}^{*} \mathrm{M}$. Since $\vartheta$ is closed (i.e. $d \vartheta=0$ ), non degenerate 2 -form on $\mathrm{T}^{*} \mathrm{M}, \vartheta$ is called as the canonic symplectic structure on $\mathrm{T}^{*} \mathrm{M}$. The cotangent bundle $\mathrm{T}^{*} \mathrm{M}$ with the symplectic structure $\vartheta$ is called as a symplectic manifold [1]. A vector field $X_{H}: T^{*} M \rightarrow T T^{*} M$ is called as Hamilton vector field if there is a function $H: T^{*} M \rightarrow R$ such that $i_{X_{H}} \vartheta=d H$. The condition $i_{X_{H}} \vartheta=d H$ is equivalent to $\vartheta\left(X_{H}, Y\right)=d H(Y)$ for $Y \in T T^{*} M$. The local expression of the Hamilton vector field is

$$
X_{H}=\frac{\partial H}{\partial p_{i}} \frac{\partial}{\partial x^{i}}-\frac{\partial H}{\partial x^{i}} \frac{\partial}{\partial p_{i}}
$$

with respect to the induced coordinates $\left(x^{i}, p_{i}\right) ; i=1, \ldots, n$ on $T^{*} M[1]$.

The curve $\phi: t \rightarrow\left(x^{i}(t), p_{i}(t)\right)$ is called as the integral curve of the Hamilton vector field $X_{H}$ since the equality $X_{H}(\phi(t))=\dot{\phi}(t)$ provides. The condition $X_{H}(\phi(t))=\dot{\phi}(t)$ is equivalent to

$$
\frac{d x^{i}}{d t}=\frac{\partial H}{\partial p_{i}}, \quad \frac{d p_{i}}{d t}=-\frac{\partial H}{\partial x^{i}} .
$$

The equations in (2.4) are called as Hamilton equations. The solution curves of these $2 \mathrm{n}$ first order differential equations describe a symplectic transformation called as phase flow in phase space [1]. The Hamilton equations are also obtained by the Legendre transformation of the Euler Lagrange equations described as follows:

$$
\frac{d}{d t}\left(\frac{\partial L}{\partial y^{i}}\right)-\frac{\partial L}{\partial x^{i}}=0
$$

where $y^{i}=\frac{d x^{i}}{d t}$. Legendre transformation is differentiable transformation from $\mathrm{TM}$ to $\mathrm{T}^{*} \mathrm{M}$ defined by

$$
£:\left(x^{i}, y^{i}\right) \rightarrow\left(x^{i}, p_{i}=\frac{\partial L}{\partial y^{i}}\right) .
$$

There is a relation between $\mathrm{H}$ and $\mathrm{L}$ as follows:

$$
H\left(x^{i}, p_{i}\right)=p_{i} y^{i}-L\left(x^{i}, y^{i}\right),
$$

where $\mathrm{L}$ is a differentiable function on TM called as Lagrangian with local expression as follows:

$$
L\left(x^{i}, y^{i}\right)=\frac{1}{2} g_{i j}(x) y^{i} y^{j}
$$

The Lagrangian of all particles in the system is equal to the difference between kinetic and potential energies of these particles $[1,7]$. 
The Hamiltonian $\mathrm{H}$ is a differentiable function on $T^{*} M$ given in (2.2). Let $\phi$ be a curve in $\mathrm{T}^{*} \mathrm{M}$. The curve $\gamma$ is called as the projected curve of $\phi$ by canonic projection $\pi$ from $T^{*} M$ to M i.e. $\pi \circ \phi=\gamma$. If the curve $\phi$ is an integral curve of the Hamilton vector field $X_{H}$, the curve $\gamma$ must be geodesic [1]. In other words, the particles in the system exposed gravitational field act along geodesic curves in the configuration space [8].

The sum of kinetic and potential energies of each of the moving particles by the only effect of the gravitational field is equal to Hamiltonian $\mathrm{H}$ which has constant value along the integral curves of the Hamilton vector field. In other words, the Hamiltonian must be constant on every point of the curve $\phi$ as the particles act along the integral curve of the Hamilton vector field. In classical mechanic, this fact is known as the conservation of energy [1].

Since the Hamiltonian $\mathrm{H}$ is constant along the integral curves of the Hamilton vector field, the Lagrangian $\mathrm{L}$ to be real valuable a function on the tangent bundle TM of the configuration space M must be constant along the integral curve of a vector field called as geodesic spray, which is the horizontal vector field in TTM [1].

The Lagrangian of the system with n-particles acting the only effect of the gravitational field is defined by (2.8). In addition, the momentum $p_{i}=\partial L / d y^{i}$ of each particle in the system must be constant. In classical mechanic, this fact is known as the conservation of momentum [7].

\section{The Euler Lagrange equations on $\left(T^{*} M,{ }^{C}\right)$}

In this section, the Lagrangian on $\left(T^{*} M,{ }^{C} g\right)$ is obtained and the general equations of geodesics of $\left(T^{*} M,{ }^{C} g\right)$ in terms of the Euler Lagrange equations are found.

Definition 3.1. The disjoint union of each tangent vector space at all point of $T^{*} M$ is called as the tangent bundle of $T^{*} M$ denoted by

$$
T T^{*} M=\bigcup_{\forall(p, \omega) \in T^{*} M} T_{(p, \omega)} T^{*} M
$$

Any point on $T T^{*} M$ is represented by $\left(p, \omega, \tilde{X}_{(p, \omega)}\right)$ where $\mathrm{p}$ is any point in a neighborhood $\mathrm{U}$ of $\mathrm{M}, \omega$ is a cotangent vector at a point $\mathrm{p}$ of $\mathrm{M}$ and $\tilde{X}_{(p, \omega)}$ is a tangent vector at a point $(p, \omega)$ of $T^{*} M . \tau_{T^{*} M}: T T^{*} M \rightarrow T^{*} M$ is called as the canonical projection map. Let $\tilde{\tilde{p}}=\left(p, \omega, \tilde{X}_{(p, \omega)}\right)$ be a point on $\left(\pi_{M} \circ \tau_{T^{*} M}\right)^{-1}(U) \subset T T^{*} M$. Then $\left(x^{1}, \ldots, x^{n}, p_{1}, \ldots, p_{n}, \dot{x}^{1}, \ldots, \dot{x}^{n}, \dot{p}_{1}, \ldots \dot{p}_{n}\right)$ is a the induced local coordinates of a point $\tilde{\tilde{p}}$ on $T T^{*} M$ where $\left\{x^{i}\right\}, i=1, \ldots, n$ is the local components of a point $\pi_{M} \circ \tau_{T^{*} M}(\tilde{\tilde{p}})=p$ of $\mathrm{M}$ and $\left\{p_{i}\right\}, i=1, \ldots, n$ is the local components of the cotangent vector $\omega$ providing $p_{i}=\omega\left(\frac{\partial}{\partial x^{i}}\right) .\left\{\dot{x}^{i}\right\}, i=1, \ldots, n$ is the local coordinate function of the tangent vector $\tilde{X}_{(p, \omega)}$ providing $\dot{x}^{i}=d x^{i}\left(\tilde{X}_{(p, \omega)}\right)=\tilde{X}_{(p, \omega)}\left[x^{i}\right]$ and $\left\{\dot{p}_{i}\right\}, i=1, \ldots, n$ is the local coordinate functions of the tangent vector $\tilde{X}_{(p, \omega)}$ providing $\dot{p}_{i}=d p_{i}\left(\tilde{X}_{(p, \omega)}\right)=\tilde{X}_{(p, \omega)}\left[p_{i}\right]$. Thus $T T^{*} M$ has $4 \mathrm{n}$ dimensional manifold structure.

Definition 3.2. The pseudo Riemann metric ${ }^{C} g$ is a tensor field of type $(0,2)$ in the cotangent bundle $T^{*} M$, whose components $\tilde{g}_{A B}$ are given by

$$
\tilde{g}_{A B}:\left(\begin{array}{cc}
-2 p_{a} \Gamma_{i j}^{a} & \delta_{i}^{j} \\
\delta_{j}^{i} & 0
\end{array}\right)_{2 n \times 2 n},
$$

where $A, B \in\{1, \ldots, 2 n\}$ and $i, j \in\{1, \ldots, n\}$. The line element of a pseudo Riemann metric ${ }^{C} g$ is given by

$$
C_{g}=-2 p_{a} \Gamma_{i j}^{a} d x^{i} d x^{j}+2 \delta_{i}^{j} d p_{i} d x^{j},
$$

with respect to the induced coordinates $\left(x^{i}, p_{i}\right)$ of $T^{*} M .{ }^{C} g$ is called as the Riemann extension of the symmetric affine connection $\nabla$ of $\mathrm{M}$ [11].

Theorem 3.1. The difference between kinetic and potential energies of the moving particles by the only effect of the gravitational field in the momentum phase space with a pseudo Riemann metric $C_{g}$ is given as follows:

$$
\tilde{L}\left(x^{i}, p_{i}, \dot{x}^{i}, \dot{p}_{i}\right)=-p_{a} \Gamma_{i j}^{a} \dot{x}^{i} \dot{x}^{j}+\dot{p}_{i} \dot{x}^{i}
$$

Proof. The difference between kinetic and potential energy of the moving particles by the only effect of the gravitational field at any stage $\left(\tilde{x}^{A}, \tilde{y}^{A}\right)$ on $T T^{*} M, \tilde{L}$, is calculated by

$$
\tilde{L}\left(\tilde{x}^{A}, \tilde{y}^{A}\right)=\frac{1}{2} \tilde{g}_{A B} \tilde{y}^{A} \tilde{y}^{B} ; A, B, D=1, \ldots 2 n
$$

where $g^{i k}, A=1, \ldots, 2 n$ corresponds to the local coordinates of a point $\tilde{p}=\left(x^{i}, p_{i}\right), i=1, \ldots, n$ on $T^{*} M$ and $\tilde{y}^{A}$ corresponds to the coordinate components $\left(\dot{x}^{i}, \dot{p}_{i}\right)$ of a vector field $\tilde{X}: T^{*} M \rightarrow T\left(T^{*} M\right), \tilde{X}=\tilde{X}^{A} \frac{\partial}{\partial \tilde{x}^{4}}$ such that $d \tilde{x}^{i}[\tilde{X}]=\dot{x}^{i}, d \tilde{x}^{n+i}[\tilde{X}]=\dot{p}_{i}$. The Lagrangian $\tilde{L}$ is a differentiable function from $T T^{*} M$ to IR. The local expression of $\tilde{L}$ is

$$
\tilde{L}\left(x^{i}, p_{i}, \dot{x}^{i}, \dot{p}_{i}\right)=\frac{1}{2}\left(\begin{array}{c}
\dot{x}^{i} \\
\dot{p}_{i}
\end{array}\right)^{T}\left(\begin{array}{cc}
-2 p_{a} \Gamma_{i j}^{a} & \delta_{i}^{j} \\
\delta_{j}^{i} & 0
\end{array}\right)\left(\begin{array}{c}
\dot{x}^{j} \\
\dot{p}_{j}
\end{array}\right)=-p_{a} \Gamma_{i j}^{a} \dot{x}^{i} \dot{x}^{j}+\dot{p}_{i} \dot{x}^{i}
$$

Definition 3.3. The one with minimum arc length of among the curves given by $\tilde{\gamma}: t \in\left[t_{0}, t_{1}\right] \subset R \rightarrow\left(x^{i}(t), p_{i}(t)\right) \in T^{*} M$ is described by integral

$$
\left.\varphi(\tilde{\gamma})=\int_{t_{0}}^{t_{1}} \tilde{L}\left(x^{i}(t), p_{i}(t), \dot{x}^{i}, \dot{p}_{i}\right)\right) d t
$$

where $\varphi(\tilde{\gamma})$ is called as functional. 
Theorem 3.2. A curve $\tilde{\gamma}: t \rightarrow\left(x^{i}(t), p_{i}(t)\right)$ in $\left(T^{*} M,{ }^{C} g\right)$ is geodesic iff $\tilde{\gamma}$ satisfies the Euler-Lagrange equations given by

$$
\frac{d}{d t}\left(\frac{\partial \tilde{L}}{\partial \dot{x}^{i}}\right)-\frac{\partial \tilde{L}}{\partial x^{i}}=0, \quad \frac{d}{d t}\left(\frac{\partial \tilde{L}}{\partial \dot{p}_{i}}\right)-\frac{\partial \tilde{L}}{\partial p_{i}}=0,
$$

where $\tilde{L}$ is defined as (3.4).

Proof. By solving the differential equations in (3.6), we get the general equations of geodesics of $T^{*} M$ with the pseudo Riemann metric ${ }^{C} g$ as follows:

$$
\begin{aligned}
& \frac{d \dot{x}^{h}}{d t}+\Gamma_{i j}^{h} \dot{x}^{i} \dot{x}^{j}=0, \\
& \frac{d \dot{p}_{h}}{d t}+p_{a} \frac{\partial \Gamma_{i j}^{a}}{\partial x^{h}} \dot{x}^{i} \dot{x}^{j}-2 p_{a} \frac{\partial \Gamma_{h j}^{a}}{\partial x^{i}} \dot{x}^{j} \dot{x}^{i}-2 \Gamma_{h j}^{a} \dot{p}_{a} \dot{x}^{j}+2 p_{a} \Gamma_{h k}^{a} \Gamma_{j i}^{k} \dot{x}^{j} \dot{x}^{i}=0,
\end{aligned}
$$

which is also obtained by classical method in Yano and Ishihara's book [11].

\section{Geodesics on $H_{1}^{2}$}

In this section, the space-like geodesics on pseudo hyperbolic 2-space are obtained by using the Euler Lagrange differential equations.

Definition 4.1. Non-degenerate, symmetric, bilinear form g is called as a semi Riemann metric in semi-Euclidean space $E_{1}^{3}$ and g is defined by

$$
g(u, v)=-u_{1} v_{1}+u_{2} v_{2}+u_{3} v_{3},
$$

for any vectors $u, v \in E_{1}^{3}$.

Definition 4.2. $H_{1}^{2}$ is a surface in $E_{1}^{3}$ given by

$$
H_{1}^{2}=\left\{u=\left(x_{1}, x_{2}, x_{3}\right):\|u\|^{2}=g(u, u)=-1, u \in E_{1}^{3}\right\} .
$$

$H_{1}^{2}$ is called as pseudo hyperbolic 2-space. $H_{1}^{2}$ may be considered as hyperboloid of two sheet in Euclidean space.

The representation of $H_{1}^{2}$ with respect to Cartesian coordinate system is given as follows:

$$
-x_{1}^{2}+x_{2}^{2}+x_{3}^{2}=-1
$$

The parametric representation of $H_{1}^{2}$ is given as follows:

$$
\begin{aligned}
& x_{1}(a, \theta)=\cosh a, \\
& x_{2}(a, \theta)=\sinh a \sin \theta, \\
& x_{3}(a, \theta)=\sinh a \cos \theta .
\end{aligned}
$$

Any curve on the surface $H_{1}^{2}$ is described by giving the following coordinates as functions of a single parameter t

$$
\begin{aligned}
& a=a(t) \\
& \theta=\theta(t) .
\end{aligned}
$$

Theorem 4.1. The length between infinitely close two points on $H_{1}^{2}$ is determined by the following metric:

$$
d s^{2}=d a^{2}+\sinh ^{2} a d \theta^{2} .
$$

Proof. In order to find length of a one parameter curve in $H_{1}^{2}$, we use the covariant differentiations of $x_{1}, x_{2}, x_{3}$ as follow:

$$
\begin{aligned}
& d x_{1}=\sinh a d a \\
& d x_{2}=\cosh a \sin \theta d a+\sinh a \cos \theta d \theta \\
& d x_{3}=\cosh a \cos \theta d a-\sinh a \sin \theta d \theta .
\end{aligned}
$$

The length between infinitely close two points on $H_{1}^{2}$ is calculated with

$$
\begin{aligned}
& d s^{2}=g\left(\left(d x_{1}, d x_{2}, d x_{3}\right),\left(d x_{1}, d x_{2}, d x_{3}\right)\right) \\
& =-d x_{1}^{2}+d x_{2}^{2}+d x_{3}^{2} .
\end{aligned}
$$

By using the covariant differentiations of $x_{1}, x_{2}, x_{3}$ in (4.4), we get

$$
d s^{2}=d a^{2}+\sinh ^{2} a d \theta^{2}
$$

and also the matrix representation of this metric is given as follows:

$$
g_{i j}:\left(\begin{array}{cc}
1 & 0 \\
0 & \sinh ^{2} a
\end{array}\right)
$$


Definition 4.3. A differentiable function from $T H_{1}^{2}$ to $R$ is defined as follows:

$$
L(a, \theta, \dot{a}, \dot{\theta})=\frac{1}{2}\left(\dot{a}^{2}+\sinh ^{2} a \dot{\theta}^{2}\right)
$$

where $\mathrm{L}$ is called as Lagrangian of $H_{1}^{2}$.

Definition 4.4. The one with minimum arc length of among the curves $\gamma$ in $H_{1}^{2}$ is described by

$$
\varphi(\gamma)=\int_{t_{0}}^{t_{1}} L(a, \theta, \dot{a}, \dot{\theta}) d t,
$$

where $\varphi(\gamma)$ describes a map from family of curves passing through different two point in $H_{1}^{2}$ to real numbers and $\gamma$ is also a curve such that $\varphi(\gamma)$ has minimum arc length on $H_{1}^{2} . \varphi(\gamma)$ is called as functional. To find $\gamma$, the Euler-Lagrange equations are used.

Definition 4.5. The trajectories of moving test particles on $H_{1}^{2}$ are determined by the following equations:

$$
\begin{aligned}
& \frac{d}{d t}\left(\frac{\partial L}{d \dot{a}}\right)-\frac{\partial L}{d a}=0, \\
& \frac{d}{d t}\left(\frac{\partial L}{d \dot{\theta}}\right)-\frac{\partial L}{d \theta}=0 .
\end{aligned}
$$

These equations are called as the Euler Lagrange equations in $H_{1}^{2}$. The particular solution providing the initial value of differential equations in (4.12) and (4.13) is a geodesic $\gamma$ passing through initial point $\left(a\left(t_{0}\right), \theta\left(t_{0}\right)\right)$ and the end point $\left(a\left(t_{1}\right), \theta\left(t_{1}\right)\right)$. The curve $\gamma$ may be visualized as the trajectory of moving a test particle by the effect of gravitational field on throat of hyperboloid of two sheets.

Definition 4.6. The line element of $H_{1}^{2}$ is given by

$$
d s^{2}=(\dot{a})^{2}+\sinh ^{2} a(\dot{\theta})^{2}=\varepsilon .
$$

The curve connecting different two point to be infinitely close on $H_{1}^{2}$ is called as the space-like curve of $H_{1}^{2}$ for $\varepsilon=1$.

Theorem 4.2. The general equation of geodesics on $H_{1}^{2}$ is given by

$$
\frac{d a}{d \theta}=\frac{\sqrt{\varepsilon \sinh ^{4} a-k_{1}^{2} \sinh ^{2} a}}{k_{1}} .
$$

Proof. In order to obtain the general equation of geodesics, we should consider the Euler Lagrange equations in (4.12), (4.13) together with metric on $H_{1}^{2}$. From the solving of the differential equations in (4.13), we obtain

$$
\frac{d}{d t}\left(\dot{\theta} \sinh ^{2} a\right)=0 \Rightarrow \dot{\theta}=\frac{k_{1}}{\sinh ^{2} a}, \mathrm{k}_{1}-\text { real constant. }
$$

If we put the value of $\dot{\theta}$ in (4.16) into at (4.14)

$$
\left(\frac{d a}{d t}\right)^{2}+\sinh ^{2} a\left(\frac{d \theta}{d t}\right)^{2}=\varepsilon \Rightarrow\left(\frac{d a}{d \theta} \frac{d \theta}{d t}\right)^{2}+\sinh ^{2} a\left(\frac{d \theta}{d t}\right)^{2}=\varepsilon
$$

we can obtain the general equation of geodesics on $H_{1}^{2}$ as follow:

$$
\frac{d a}{d \theta}=\frac{\sqrt{\varepsilon \sinh ^{4} a-k_{1}^{2} \sinh ^{2} a}}{k_{1}} .
$$

Since the gradient vector field to be normal to tangent vector space at any point of $H_{1}^{2}$ is time like, $H_{1}^{2}$ must be called as a space like surface. So, $H_{1}^{2}$ has only space like geodesics. The general equation of these geodesics on $H_{1}^{2}$ is given by the following theorem.

Theorem 4.3. The space-like geodesics on $H_{1}^{2}$ are given by the following equations:

$$
\frac{\sqrt{1-k_{1}^{2} \operatorname{cosech}^{2} a}}{\sqrt{k_{1}^{2}+1}}=\frac{\sin \theta}{k_{2}}, \frac{x_{1}^{2}}{k_{1}^{2}+1}-\frac{x_{2}^{2}}{k_{2}^{2}}=1
$$

with respect to generalized and cartesian coordinates.

Proof. If we chose $\varepsilon=1$ in (4.15), we can obtain following surface:

$$
\frac{\sqrt{1-k_{1}^{2} \operatorname{cosech}^{2} a}}{\sqrt{k_{1}^{2}+1}}=\frac{\sin \theta}{k_{2}}, \frac{x_{1}^{2}}{k_{1}^{2}+1}-\frac{x_{2}^{2}}{k_{2}^{2}}=1
$$

with respect to generalized coordinates $(a, \theta)$ and cartesian coordinates.

The space-like geodesic characterized by trajectory of a moving test particle by the effect of gravitational field acts faster than speed of light on the surface $H_{1}^{2}$. This mechanical interpretation was inspired from [9]. 


\section{Geodesics on $\left(\mathbf{T}^{*} \mathbf{H}_{1}^{2}, \mathrm{~g}^{\mathrm{C}}\right)$}

In this section, the general equations of geodesics on the cotangent bundle of pseudo hyperbolic 2-space with the pseudo Riemann metric ${ }^{C} g$ are calculated.

Definition 5.1. Let $H_{1}^{2}$ be pseudo hyperbolic 2-space and $T^{*} H_{1}^{2}$ be its cotangent bundle. As any point q on $H_{1}^{2}$ has the generalized coordinates $(a, \theta)$, the point $\left(q, \eta_{q}\right)$ on $T^{*} H_{1}^{2}$ has the local coordinates $\left(a, \theta, p_{1}, p_{2}\right)$ where $p_{1}, p_{2}$ are the local coordinate function of the cotangent vector $\eta_{q}$ at $q$. In addition, the point $\left(q, \eta_{q}, X_{\left(q, \eta_{q}\right)}\right)$ on $T T^{*} H_{1}^{2}$ has the local coordinates $\left(a, \theta, p_{1}, p_{2}, \dot{a}, \dot{\theta}, \dot{p}_{1}, \dot{p}_{2}\right)$ and the point $\left(q, \eta_{q}, \omega_{\left(q, \eta_{q}\right)}\right)$ on $T^{*} T^{*} H_{1}^{2}$ has the local coordinates $\left(a, \theta, p_{1}, p_{2}, \omega_{a}, \omega_{\theta}, \omega_{p_{1}}, \omega_{p_{2}}\right)$ where $\omega_{a}, \omega_{\theta}, \omega_{p_{1}}, \omega_{p_{2}}$ are the local coordinate functions of the cotangent vector at $\left(q, \eta_{q}\right)$ i.e. $\omega_{\left(q, \eta_{q}\right)} \in T_{\left(q, \eta_{q}\right)}^{*} T^{*} H_{1}^{2}$.

Theorem 5.1. The general equations of geodesics of $H_{1}^{2}$ are represented by the following equations:

$$
\ddot{a}-\cosh a \sinh a(\dot{\theta})^{2}=0, \ddot{\theta}+2 \operatorname{coth} a \dot{a} \dot{\theta}=0 .
$$

Proof. To find the general equations of geodesics on $H_{1}^{2}$, we need to the general formula of the geodesic equations on Riemann manifolds given by

$$
\ddot{x}^{\alpha}+\Gamma_{\gamma \beta}^{\alpha} \dot{x}^{\gamma} \dot{x}^{\beta}=0,
$$

and the Christoffel symbols given by

$$
\Gamma_{\beta \gamma}^{\alpha}=\frac{1}{2} g^{\alpha \mu}\left(g_{\mu \gamma, \beta}+g_{\beta \mu, \gamma}-g_{\beta \gamma, \mu}\right),
$$

and where $g_{\alpha \beta}$ is the components of the matrix representation of the metric tensor of $H_{1}^{2}$ and $g^{\alpha \beta}$ is inverse of $g_{\alpha \beta}$. The non-zero components of the Christoffel symbols of $H_{1}^{2}$ are obtained as follows:

$$
\Gamma_{22}^{1}=-\sinh a \cosh a, \quad \Gamma_{12}^{2}=\Gamma_{21}^{2}=\operatorname{coth} a .
$$

If we put the non-zero Christoffel symbols in (5.3) into (5.1), we get the general equations of geodesics on $H_{1}^{2}$ as follows:

$$
\ddot{a}-\cosh a \sinh a(\dot{\theta})^{2}=0, \ddot{\theta}+2 \operatorname{coth} a \dot{a} \dot{\theta}=0 .
$$

Theorem 5.2. The pseudo Riemann metric ${ }^{C} g$ on $T^{*} H_{1}^{2}$ has components

$$
C_{g}=-4 p_{2} \operatorname{coth} a d a d \theta+2 p_{1} \sinh a \cosh a(d \theta)^{2}+2 d a d p_{1}+2 d \theta d p_{2} .
$$

Proof. By using the equations in (3.3) and (5.3), the pseudo Riemann metric ${ }^{C} g$ on $T^{*} H_{1}^{2}$ has following component

$$
C_{g}=\left(-2 p_{1} \Gamma_{11}^{1}-2 p_{2} \Gamma_{11}^{2}\right)(d a)^{2}+2\left(-2 p_{1} \Gamma_{12}^{1}-2 p_{2} \Gamma_{12}^{2}\right) d a d \theta+\left(-2 p_{1} \Gamma_{22}^{1}-2 p_{2} \Gamma_{22}^{2}\right)(d \theta)^{2}+2 d a d p_{1}+2 d \theta d p_{2} .
$$

The matrix representation of ${ }^{C} g$ is as follows:

$$
g_{A B}:\left(\begin{array}{cccc}
0 & -2 p_{2} \operatorname{coth} a & 1 & 0 \\
-2 p_{2} \operatorname{coth} a & 2 p_{1} \sinh a \cosh a & 0 & 1 \\
1 & 0 & 0 & 0 \\
0 & 1 & 0 & 0
\end{array}\right),
$$

and the matrix representation of inverse of ${ }^{C} g$ is as follows:

$$
\left({ }^{C} g\right)^{-1}:\left(\begin{array}{cccc}
0 & 0 & 1 & 0 \\
0 & 0 & 0 & 1 \\
1 & 0 & 0 & 2 p_{2} \operatorname{coth} a \\
0 & 1 & 2 p_{2} \operatorname{coth} a & -2 p_{1} \sinh a \cosh a
\end{array}\right)
$$

Theorem 5.3. The non-zero components of the Christoffel symbols of $T^{*} H_{1}^{2}$ with the pseudo Riemann metric $C_{g}$ has the following components:

$$
\begin{aligned}
& \Gamma_{22}^{1}=-\sinh a \cosh a \\
& \Gamma_{12}^{2}=\Gamma_{21}^{2}=\operatorname{coth} a \\
& \Gamma_{12}^{3}=\Gamma_{21}^{3}=2 p_{2} \operatorname{coth}^{2} a, \Gamma_{22}^{3}=-p_{1} \cosh 2 a, \Gamma_{24}^{3}=\Gamma_{24}^{3}=-\operatorname{coth} a \\
& \Gamma_{11}^{4}=2 p_{2} \operatorname{cosech} a, \Gamma_{12}^{4}=\Gamma_{21}^{4}=-p_{1}, \Gamma_{22}^{4}=-2 p_{2} \cosh ^{2} a, \\
& \Gamma_{14}^{4}=\Gamma_{41}^{4}=-\operatorname{coth} a, \Gamma_{23}^{4}=\Gamma_{32}^{4}=\sinh a \cosh a .
\end{aligned}
$$


Proof. By using the general formula of the Christoffel symbols in (5.2), it is seen to be correct of theorem.

Theorem 5.4. Any curve $\tilde{c}: t \rightarrow \tilde{c}(t)=\left(a, \theta, p_{1}, p_{2}\right)$ on $\left(T^{*} H_{1}^{2}, C_{g}\right)$ is geodesic iff following differential equations system must provide:

$$
\begin{aligned}
& \ddot{a}-\sinh a \cosh a(\dot{\theta})^{2}=0, \\
& \ddot{\theta}+2 \operatorname{coth} a \dot{a} \dot{\theta}=0, \\
& \ddot{p}_{1}+4 p_{2} \operatorname{coth}^{2} a \dot{a} \dot{\theta}-p_{1} \cosh 2 a(\dot{\theta})^{2}-2 \operatorname{coth} a \dot{\theta} \dot{p}_{2}=0, \\
& \ddot{p}_{2}+2 p_{2} \operatorname{cosech}{ }^{2} a(\dot{a})^{2}-2 p_{1} \dot{a} \dot{\theta}-2 p_{2} \cosh ^{2} a(\dot{\theta})^{2}-2 \operatorname{coth} a \dot{a} \dot{p}_{2}+2 \sinh a \cosh a \dot{\theta} \dot{p}_{2}=0 .
\end{aligned}
$$

Proof. By using the general formula of the geodesic equation in (5.1) and components of the Christoffel symbol in (5.6), it is seen easily to be correct of theorem.

Theorem 5.5. The general equations of geodesics of $\left(T^{*} H_{1}^{2},{ }_{g}\right)$ obtained in Theorem 3.2 by using the Euler Lagrange equations provide the geodesic equations obtained by classical method at Theorem 5.1.

Proof. If we rewrite with respect to the components of the general equations of geodesics of $\left(T^{*} M,{ }^{C} g\right)$ obtained by Theorem 3.2 for $\left(T^{*} H_{1}^{2}, C_{g}\right)$, we get the following equations:

$$
\begin{aligned}
& \frac{d \dot{x}^{1}}{d t}+\Gamma_{22}^{1} \dot{x}^{2} \dot{x}^{2}=0 \\
& \frac{d \dot{x}^{2}}{d t}+2 \Gamma_{12}^{1} \dot{x}^{1} \dot{x}^{2}=0 \\
& \frac{d \dot{p}_{1}}{d t}+p_{1} \frac{\partial \Gamma_{22}^{1}}{\partial x^{1}} \dot{x}^{2} \dot{x}^{2}-2 \Gamma_{12}^{2} \dot{p}_{2} \dot{x}^{2}+2 p_{2} \Gamma_{12}^{2} \Gamma_{21}^{2} \dot{x}^{1} \dot{x}^{2}=0 \\
& \frac{d \dot{p}_{2}}{d t}-2 p_{2} \frac{\partial \Gamma_{21}^{2}}{\partial x^{1}} \dot{x}^{1} \dot{x}^{1}-2 p_{1} \frac{\partial \Gamma_{22}^{1}}{\partial x^{1}} \dot{x}^{2} \dot{x}^{1}+2 p_{1} \Gamma_{22}^{1} \Gamma_{12}^{2} \dot{x}^{2} \dot{x}^{1}+2 p_{2} \Gamma_{21}^{2} \Gamma_{22}^{1} \dot{x}^{2} \dot{x}^{2}-2 \Gamma_{21}^{2} \dot{p}_{2} \dot{x}^{1}-2 \Gamma_{22}^{1} \dot{p}_{1} \dot{x}^{2}=0
\end{aligned}
$$

When we use the coefficients of the Christoffel symbol in (5.1), it is seen to be the above equations is equal to the geodesic equations obtained by classical method at Theorem 5.4.

\section{Conclusion}

This study contains six sections. In the second section, the geometrical interpretation of the mechanic concepts are considered such as the movement of the particles by the effect of the gravitational field, the conservation of momentum or energy, well known in classic mechanic. In the third section, the general equations of geodesics on the cotangent bundle $\left(\mathrm{T}^{*} \mathrm{H}_{1}{ }^{2}, \mathrm{~g}^{\mathrm{C}}\right)$ are found by using the Euler Lagrange equations. In the fourth section, the space-like geodesics on pseudo hyperbolic 2-space are obtained by using the Euler Lagrange equations.

Finally, in the fifth section, the general equations of geodesics on the cotangent bundle of pseudo hyperbolic 2 -space $\left(\mathrm{T}^{*} \mathrm{H}_{1}{ }^{2}, \mathrm{~g}^{\mathrm{C}}\right)$ are obtained by using general formula of geodesics on a Riemann manifold and then it is shown that the general equations of geodesics on $\left(\mathrm{T}^{*} \mathrm{H}_{1}{ }^{2}, \mathrm{~g}^{\mathrm{C}}\right)$ are equal to the geodesic equations obtained by using the Euler Lagrange equations in the second section.

\section{References}

[1] R. Abraham and J. E. Marsden, Foundations of mechanics, W. A. Benjamin Inc.,New York, 1967.

[2] I. Ayhan, On the tangent sphere bundle of the pseudo hyperbolic two space, Global Journal of Advanced Research on Classical and Modern Geometries, vol. 3, no. 2, pp 76-90, 2014.

[3] I. Ayhan, On the sphere bundle with the Sasaki semi Riemann metric of a space form, Global Journal of Advanced Research on Classical and Modern Geometries, vol. 3, no. 1, pp 25-35, 2014.

[4] I. Ayhan, Geodesics on the tangent sphere bundle of 3-Sphere, International Electronic Journal of Geometry, vol. 6, no. 2, pp 100-109, 2013.

[5] A. C. Coken, I Ayhan, On the geometry of the movements of the particles in a Hamilton space, Abstract and Applied Analysis, DOI:10.1155/2013/830147, 2013.

[6] P. Free, Introduction to general relativity, Lecture Notes, Virgo site, 2003.

[7] R. Miron, H. Hrimiuc, H. Shimada, and V. S. Sabau, The geometry of Hamilton and Lagrange spaces, Kluwer Academic, New York, USA, 2001.

[8] A. Polnarev, Motion of a test particle in a gravitational field and Hamilton Jacobi equations, relativity and gravitation, Lectures Notes 5, 2011.

[9] S. Waner, G. C. Levine, Introduction to differential geometry and general relativity, Lectures Notes, 2005.

[10] C. K. Wonk, Classical physics in Galilean and Minkowski space-times, Lecture Notes 3, 2009.

[11] K. Yano and S. Ishihara, Tangent and cotangent bundles, Marcel Decker Inc., New York, 1973. 